\title{
Extragalactic Survey of UV Galaxies Using GALEX
}

\author{
Lakshmi S. Bose ${ }^{1}$, K. Narayanankutty ${ }^{1}$, N. V. Sujatha ${ }^{2}$ \& Jayant Murthy ${ }^{3}$ \\ ${ }^{1}$ Amrita Vishwavidya Peetham, Amritapuri Campus, Kollam. \\ ${ }^{2}$ St. Xavier's College for Women, Aluva. \\ ${ }^{3}$ Indian Institute of Astrophysics, Bangalore.
}

\begin{abstract}
We report here the results of the study of extragalactic radiation using number counts of galaxies at high galactic latitudes. For this study nine GA LEX DIS targets around ELAISNI in the North Pole. The overlapped regions of two missions cover an area of $\sim 2$ square degrees in the selected region. By comparing the point source catalogs of GALEX and Spitzer, we obtained the UV flux (both FUV \& NUV) in magnitude for each matching galaxy. We then calculated the number counts of galaxies, $N$ Objects/square degree/magnitude, from the region and compared it with the existing values in the literature. There is drop out of galaxies at the fainter end which is due to low resolution and sensitivity of GALEX when we compared it with Spitzer Extragalactic Representative Volume Survey (SERVS) region - ELIAS-N1 - centered at $16^{h} 10^{m} 01^{s}$, $+54^{\circ} 30^{\prime} 36^{\prime \prime}$. From this study we could establish the presence of unresolved objects in the GALEX field.
\end{abstract}

Keywords: GALEX, UV Number counts, UV magnitude, Extragalactic survey, resolved galaxies, unresolved objects

\section{Introduction}

The number counts of detected galaxies from two wavelength bands can provide an open scenario of extragalactic contribution. The Pegasus launched Galaxy Evolution Explorer (GALEX) in 2003 under NASA's Small Explorer (SMEX) is the first Ultraviolet (UV) mission to conduct two imaging surveys in far UV band (FUV:135 nm-180nm) and near UV band (NUV:180-200 nm) with significant spatial resolution of 3"- 6".Tthe working of this satellite were narrated by D. C. Martin et al. 2005 [1] \& Morrissey et al. 2007 [2]. Analysis of GALEX observations at high galactic latitude is momentous for understanding more about the extragalactic light and its contribution in the UV sky.

There has been significant work on number counts carried out in UV band (Table 1). Measuring the number counts of field galaxies within an observed area as a function of the magnitude of galaxies is one of the fundamental techniques used for the study of extragalactic light, galaxy evolution throughout cosmic time and can be used to test theoretical models of galactic evolution. Further, the changes in the slope of number count distributions reflectphysicalchanges in theunderlyinggalaxy populations.

Table 1

\begin{tabular}{|c|c|c|c|c|}
\hline Author & Instrument & Wavelength & Area covered & $\begin{array}{c}\text { Magnitude } \\
\text { limits }\end{array}$ \\
\hline Millard et al. (1992)[3] & Balloon borne Telescope & $2000 \AA$ & 6 square degree & $15.0-18.5$ \\
\hline Gardner et al. (2000)[4] & Hubble Space Telescope & $\begin{array}{c}\text { NUV-2365 } \\
\text { FUV- } 1595 \AA\end{array}$ & $1.54 \operatorname{arcmin}^{2}$ & $24.5-29.5$ \\
\hline $\begin{array}{c}\text { Teplitz et al. (2006)[5] } \\
\text { Xu et al. } \\
(2005)[6]\end{array}$ & Hubble Space Telescope & $1600 \AA$ & $3.77 \operatorname{arcmin}^{2}$ & $20.5-28.5$ \\
\hline
\end{tabular}

\section{Observations}

In this study, 9 GALEX deep imaging surveys (DIS) catalogs were taken from the North Polar Region. Details are given in the Table 2 . These targets are from an optically thin region. The dust $(\mathrm{E}(\mathrm{B}-\mathrm{V}))$ variation in the field is $0.01-0.05$, which corresponds to an optical depth of $0.0812-0.406$ (Schlegel 1998) [7]. The standard GALEX pipeline (Morrissey et al. 2007) provides a catalog of UV sources based on the detection and flux measurements with SExtractor (Bertin \& Arnouts, 1996)[11] for each flux calibrated image but, given the GALEX point spread function of 4.5 and 5.4 arcsec in the FUV and NUV bands, respectively. The data files 
used in the study were in Flexible Image Transport System (FITS: Well et al. 1981)[8]. These images contain both point sources and diffuse background components. Here the diffuse back ground is subtracted and the edge effects were eliminated by the usage of central $0.55^{\circ}$ from $1.25^{\circ}$ GALEX field of view.

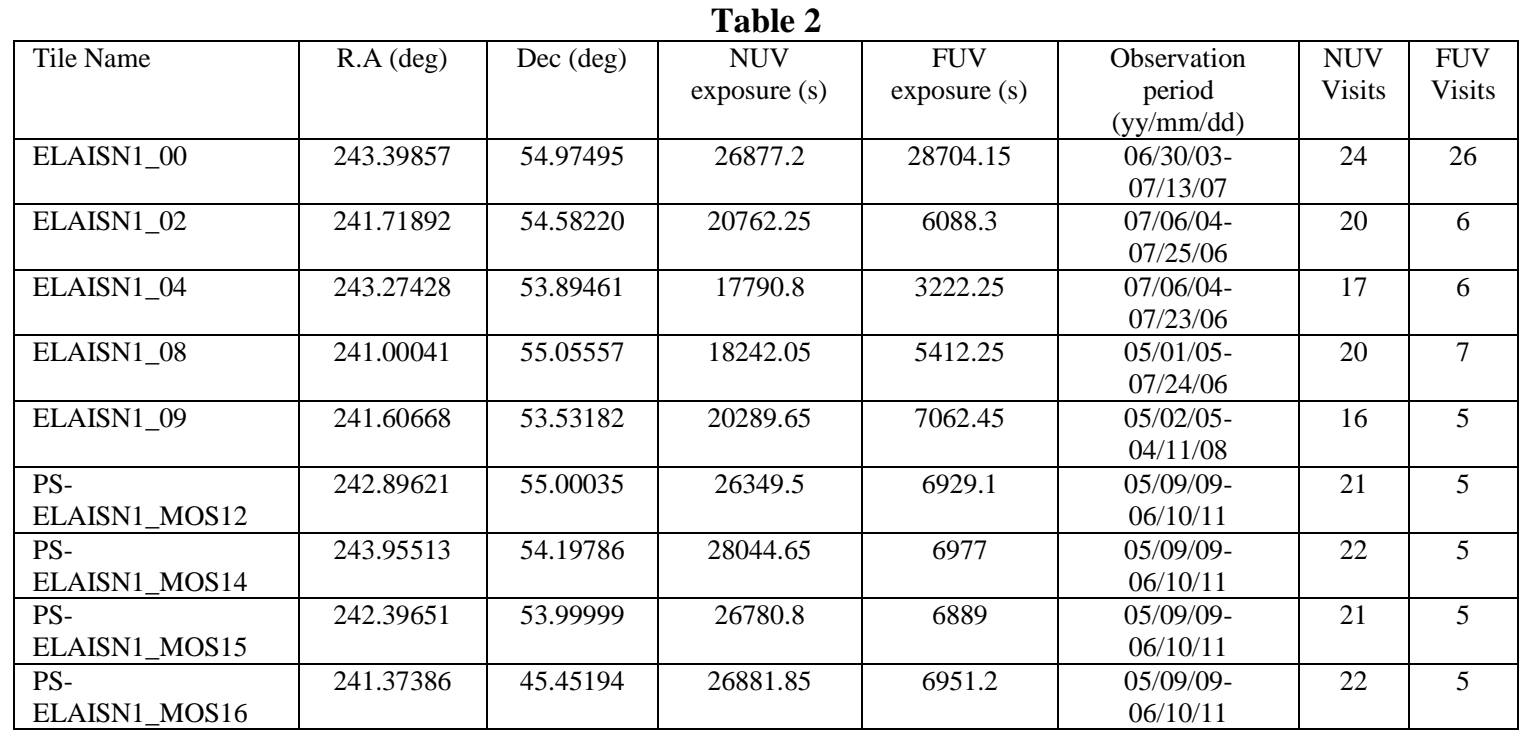

\section{Number Counts Of Galaxies}

There are totally 37,548 galaxies in this field and the stars are removed from the catalog by using the prescription of Groenewegen et al. (2002)[9] and Fadda et al. (2004)[10] based on the stellarity index value (CLASS_STAR) given below:

If Class Star $>0: 85$ For $R<23$;

Class Star > 0:90 Otherwise:

The GALEX photometric catalog produced using SExtractor, extracted the flux details in CPS for the matching objects. The flux is then converted into $\mathrm{AB}$ magnitude as follows

Fuv: $M_{a b}=-2.5 X \log _{10}(C p s)+18.82$

Nuv: $M_{a b}=-2.5 \times \log _{10}(C p s)+20.08$

The duplicate objects obtained from the field due to the overlapping of GALEX FOV are removed carefully. Number counts of galaxies, $\log \left(\mathrm{N} / \mathrm{sq} \cdot \mathrm{deg}^{2} / \mathrm{mag}\right.$ ) for FUV \& NUV, from GALEX ELAISN1 region are plotted against $\mathrm{AB}$ magnitude in Fig 2 and 3. The FUV and NUV magnitude range for the objects in the field is 17.5-26.5. The 'square box' represents the results of Xu et al (2005)[6] who measured the bright FUV and NUV galaxy counts between magnitudes 14 and 23.8 using 36 Medium depth Survey fields and 3 Deep Survey fields obtained with GALEX.

Our results are in good agreement with that of Xu et al. (2005) up to the level of magnitude less than 22 because DIS observations of GALEX have several thousands of second's exposure time and hence the data are $80 \%$ complete to NUV magnitude of 23 and FUV magnitude of 23.5, but the source confusion is significant in these observations. There is a drop out of number counts of galaxies occurs using GALEX at fainter side. 


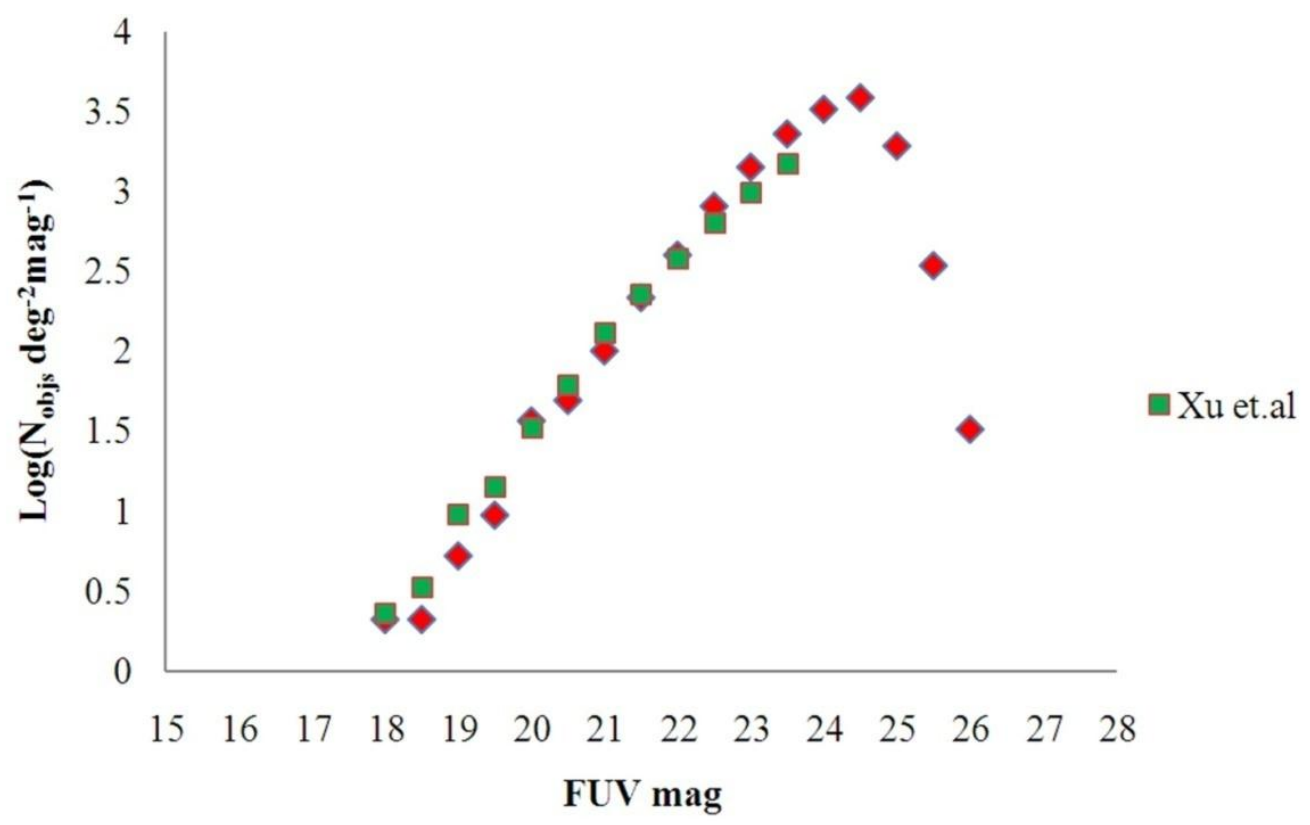

Fig 2 Number counts as a function of FUV magnitude

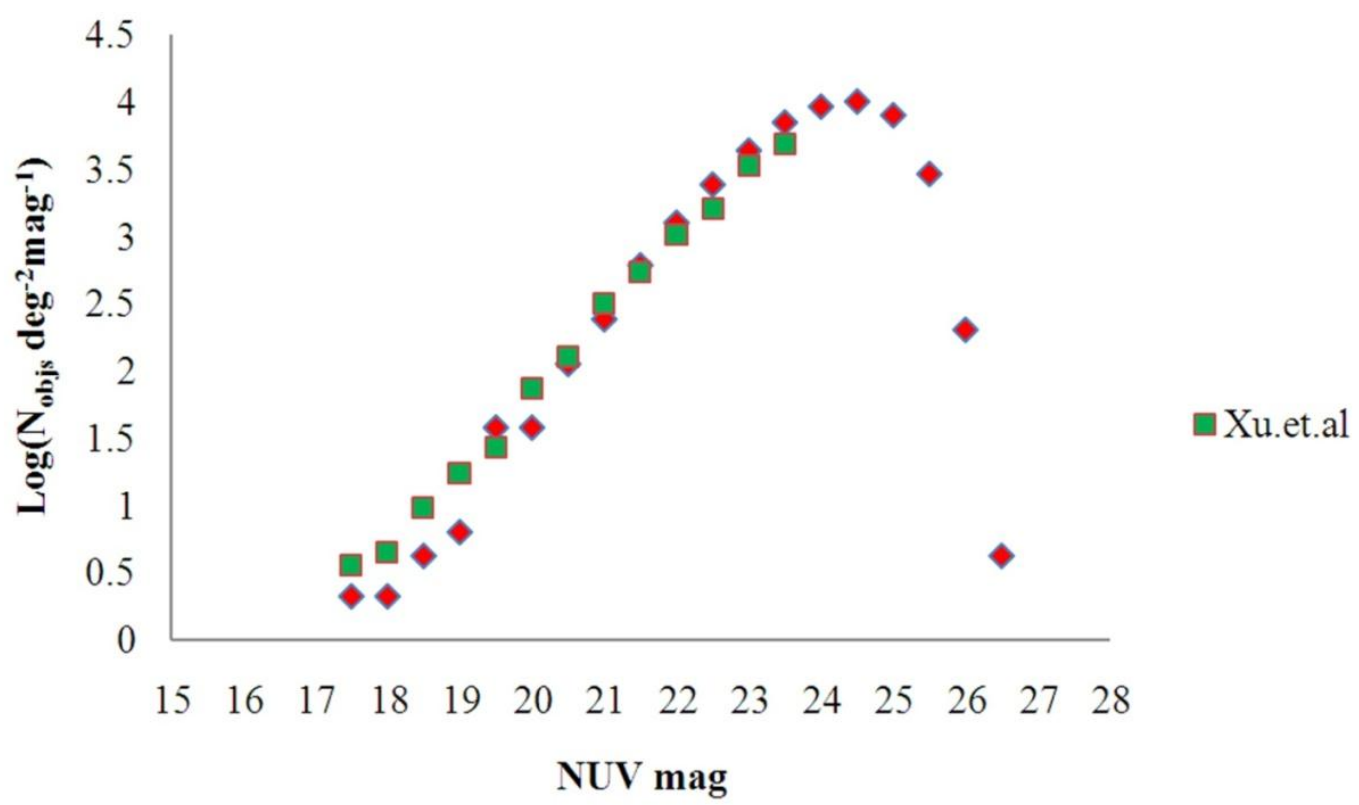

Fig 3 Number counts indicates a drop out at 24.5 NUV magnitude

\section{Results And Discussions}

We report here the results of preliminary study for the measurement of extragalactic radiation in the GALEX fields. The FUV and NUV number counts of galaxies estimated as a function of $\mathrm{AB}$ magnitude from the Spitzer overlapped GALEX observed region in ELAISN1. Bright UV galaxy counts between 14 and 23.8 magnitudes in FUV and NUV were measured by Xu et al. (2005) using 36 Medium depth Survey fields and 3 Deep Survey fields obtained with the GALEX from an area $\sim 20 \mathrm{deg}^{2}$. Our technique of estimation of number counts is different from that of Xu et al (2005) but the results are in good agreement with each other especially in the bright end, whereas the inconsistency/incompleteness in the faint end ( $\mathrm{AB}$ magnitude >23) is mainly due to the source confusion and magnitude compatibility with diffuse sources. The drop out of counts in the fainter side indicates low sensitivity of GALEX and also the presence of unresolved objects in the this field. 


\section{Future Work}

Spitzer spacecraft is more sensitive to galaxies than GALEX, therefore we need to identify the Spitzer positions of galaxies in GALEX fields directly, estimate the flux around each object and redo the analysis without considering the GALEX catalog derived by SExtractor. This may avoid the source confusion in the fainter magnitude side and produce better results. With this we can directly calculate the contribution of extragalactic background light (EGL) in the GALEX bands by integrating the emissions of these galaxies or by integrating the formula,

$\mathbf{I}_{\mathrm{EGL}}=\mathbf{1 0}^{-0.4(\mathrm{m0}-\mathrm{mAB}} * \mathbf{N}\left(\mathrm{m}_{\mathrm{AB}}\right)$

Where $\mathrm{m} 0$ is the zero point magnitude that corresponds to the $\mathrm{AB}$ magnitude of a $1 \mathrm{c} / \mathrm{s}$ flat-field corrected detection and $\mathrm{N}$ is the number counts. These measurements set a lower limit for the total FUV/NUV background light.

\section{Acknowledgment}

This research is based on data from the NASA's GALEX program. GALEX is operated for NASA by the California Institute of Technology under NASA contract NAS5-98034. We acknowledge the use of NASA's Astrophysics Data System and Sky View facility (http://skyview.gsfc.nasa.gov) located at NASA Goddard Space Flight Center.

[1] D. Christopher Martin, “The Galaxy Evolution Explorer: A Space Ultraviolet Survey Mission”, James Fanson, David Schiminovich „Eds., Astrophysical Journal 619, L1-L6, 2005.

[2] Patrick Morrissey," The Calibration And Data Products Of Galex", Tim Conrow, Tom A. Barlow, Eds., Astrophysical Journal Supplement Series, 173, 682, 2007

[3] Milliard B,” Galaxy Counts At Ultraviolet Wavelengths (2000a)”, Donas. J, Laget. M, Armand. C ,Eds., Astronomy \& Astrophysics, 257, 24, 1992.

[4] Gardner J, "Ultraviolet Galaxy Counts From Stis Observations Of The Hubble Deep Fields" „P.,Brown T.M., \& Ferguson. H.C., Astrophysical Journal, 252, L79, 2000.

[5] Teplitz H. I., "Far-Ultraviolet Imaging Of The Hubble Deep Field-North: Star Formation In Normal Galaxies At Z<1", Siana, B.; Brown, T. M.; Chary, R.; Colbert, J. W, Eds., Astronomical Journal, 132, 853, 2006.

[6] Xu C. K., Et Al," Number Counts Of Galex Sources In Far-Ultraviolet (1530 A) And Near-Ultraviolet (2310 A) Bands", Jose Donas, Stephane Arnouts, Eds., Astrophysical Journal, 619, L11, 2005.

[7] Schlegel, D. J.” Maps Of Dust Infrared Emission For Use In Estimation Of Reddening And Cosmic Microwave Background Radiation Foregrounds", Finkbeiner, D. P., \& Davis, M., Eds., Astrophysical Journal, 500, 525, 1998.

[8] Wells, D. C.” Fits - A Flexible Image Transport System”, Greisen, E. W. \& Harten, R. H., Eds.,, Astronomy \&Astrophysics Supplement, 44, 363. 1981.

[9] Groenewegen, M. A. T., "Eso Imaging Survey. The Stellar Catalogue In The Chandra Deep Field South ",Girardi, L.; Hatziminaoglou, E.;Benoist C, .Eds ., Astronomy \&Astrophysics, 392, 7412002.

[10] Fadda D.” The Spitzer Space Telescope First-Look Survey: Kpno Mosaic-1 R-Band Images And Source Catalogs”, Buell T.J., Ford A., Eds.,, Astrophysical Journal, 128, 15, 2004.

[11] Bertin, E.; Arnouts, S., Sextractor: Software For Source Extraction, Astronomy And Astrophysics Supplement, V.117, P.393-404 